\title{
FUNCIONALIDADE E QUEDAS EM IDOSOS INSTITUCIONALIZADOS: PROPOSTAS DE AÇÕES DE ENFERMAGEM*
}

\author{
Ariane Minussi Feliciani1 ${ }^{1}$, Silvana Sidney Costa Santos ${ }^{2}$, Rafaela Vivian Valcarenghi ${ }^{3}$
}

\begin{abstract}
RESUMO: Trata-se de uma pesquisa avaliativa, com o objetivo de propor ações de melhoria para uma Instituição de Longa Permanência para Idosos, no Rio Grande do Sul, Brasil, que auxiliem o enfermeiro na manutenção da funcionalidade e na prevenção das quedas dos residentes. Foi construída, testada e aplicada uma ferramenta avaliativa, fundamentada nas políticas públicas, para ser utilizada pelo enfermeiro. Com a ferramenta, aplicada em setembro de 2009, foi possível identificar e apresentar ações que podem contribuir para a manutenção da funcionalidade e para a prevenção de quedas. Espera-se que esta iniciativa, além de instrumentar o enfermeiro no cuidado ao idoso, promova a conexão entre o Serviço e a Academia para que outras frentes de ações estratégicas sejam articuladas e voltadas à otimização do cuidado de enfermagem ao idoso institucionalizado.
\end{abstract}

PALAVRAS-CHAVE: Enfermagem; Idoso; Acidentes por quedas, Instituição de longa permanência para idosos.

\section{FUNCTIONING AND FALLS IN THE INSTITUTIONALIZED ELDERLY: PROPOSALS FOR NURSING ACTIONS}

\begin{abstract}
This is an evaluative piece of research which aimed to propose improving actions for a long-term elderly care center in Rio Grande do Sul, Brazil, which could help the nurses to maintain residents' functioning and reduce their falls. A public-policy-based evaluative tool was built, tested and applied, for use by nurses. With this tool, first applied in September 2009, it was possible to identify and present actions which might contribute to maintaining residents' functioning and reducing falls. It is hoped that this initiative, apart from aiding the nurse in care of the elderly, may promote the connection between the workplace and higher learning so that other areas with potential for strategic actions may be identified and optimized for nursing care of the elderly residents in long-term care facilities.

KEYWORDS: Nursing; Elderly; Accidents from falls; Long-term care facilities for the elderly.

\section{FUNCIONALIDAD Y CAÍDAS EN ANCIANOS INSTITUCIONALIZADOS: PROPUESTAS DE ACCIONES DE ENFERMERÍA}

RESUMEN: Es una investigación de evaluación, con el objetivo de proponer acciones de mejoría para una Institución de Larga Permanencia para Ancianos, en Rio Grande do Sul, Brasil, que auxilíen el enfermero en la manutención de la funcionalidad y en la prevención de caídas de los residentes. Fue construida, testada y aplicada una herramienta de evaluación, fundamentada en las políticas públicas, a fin de ser utilizada por el enfermero. Con la herramienta, aplicada en septiembre de 2009, fue posible identificar y presentar acciones que pueden contribuir para la manutención de la funcionalidad y para la prevención de caídas. Se espera que esta iniciativa, además de instrumentar el enfermero en el cuidado al anciano, promueva la conexión entre el Servicio y la Academia, para que otras posibilidades de acciones estratégicas sean articuladas y direccionadas al perfeccionamiento del cuidado de enfermería al anciano institucionalizado. PALABRAS-CLAVE: Enfermería; Anciano; Accidentes por caídas; Institución de larga permanencia para ancianos.

\footnotetext{
* Artigo vinculado à Dissertação de Mestrado em Enfermagem "Construção de ferramenta avaliativa fundamentada nas políticas públicas para idosos: contribuições para o enfermeiro na prevenção de quedas”, Universidade Federal do Rio Grande - FURG, 2009.

${ }^{1}$ Enfermeira da Associação de Caridade Santa Casa do Rio Grande. Mestre em Enfermagem.

${ }^{2}$ Enfermeira. Doutora em Enfermagem. Professora da Escola de Enfermagem da FURG. Líder do Grupo de Estudo e Pesquisa em Gerontogeriatria, Enfermagem/Saúde e Educação - GEP-GERON.

${ }^{3}$ Enfermeira. Professora do Instituto Federal de Educação, Ciência e Tecnologia de Santa Catarina. Mestre em Enfermagem. Doutoranda pelo Programa de Pós-Graduação em Enfermagem da Universidade Federal de Santa Catarina.
} 


\section{INTRODUÇÃO}

Refletir sobre o processo de envelhecimento se faz relevante, visto que nas últimas décadas aumentou a população idosa mundial. Este fato é observado em países em desenvolvimento, como o Brasil, que vem mudando o seu perfil demográfico, com prognóstico de crescimento ainda maior para os próximos anos. A proporção de idosos, entre 1998 e 2008, aumentou de $8,8 \%$ para $11,1 \%$. O Rio Grande do Sul continua sendo um dos estados com maior magnitude de idosos, com $13,5 \%$ em sua população ${ }^{(1)}$.

Verifica-se que a necessidade da existência de Instituições de Longa Permanência do Idoso (ILPI) surge em função desse aumento e das dificuldades apresentadas pelos familiares no que se refere à tarefa de cuidá-los no domicílio. Acredita-se que a tendência é aumentar o número de ILPI no Brasil, ainda que as políticas públicas de saúde priorizem o cuidado prestado ao idoso pela família.

Pensadas como cenários de cuidados, as ILPI ainda se constituem em um desafio, principalmente se comparadas à proposta da promoção da saúde, que se fundamenta no empoderamento, expresso, entre outros aspectos, pelo direito à individualidade. Porém, visualizam-se pontos precursores para a mudança na maneira de cuidar do idoso em ILPI, pois até pouco tempo eram mínimas as atividades realizadas em prol dos institucionalizados ${ }^{(2)}$.

Dentre os dispositivos legais que dizem respeito ao idoso institucionalizado, seja independente ou dependente de cuidados específicos, citam-se: a Política Nacional do Idoso (PNI) - Lei n. 8842/1995 e Decreto 1948/1996 ${ }^{(3)}$; a Portaria Interministerial MS/MPAS n. 5.153 - Programa Nacional dos Cuidadores de Idosos ${ }^{(4)}$; a Lei n. 10.741/2003 - o Estatuto do Idoso(5); a RDC n. 283/2005, da Agência Nacional de Vigilância Sanitária (Anvisa) - Regulamento técnico para funcionamento das ILPI ${ }^{(6)}$.

Mesmo o idoso independente, quando institucionalizado, pode desenvolver diferentes níveis de dependência, evento relacionado à sua dificuldade de aceitação e de adaptação às novas condições de vida, bem como à falta de motivação e de encorajamento, comuns no contexto institucional $^{(7)}$. Estas são situações que necessitam ser observadas pelos profissionais atuantes nessas instituições. Estes profissionais precisam ter formação específica para as funções que desempenham e necessitam participar de educação permanente, de acordo com o contexto da instituição e o perfil dos idosos residentes. Importante, ainda, torna-se a realização de supervisão e a avaliação desses cuidados pelo enfermeiro da ILPI.

O que vem chamando a atenção dos trabalhadores da saúde e tem relação com a funcionalidade, em especial do idoso, são as quedas, evento de notificação compulsória aos órgãos sanitários competentes e que necessita ser prevenido continuamente, no contexto domiciliar, hospitalar, institucional ou em outros ambientes. Esse evento adverso refere-se a uma situação com características não intencionais, resultante da mudança na posição do corpo humano para um nível mais baixo, em relação à sua posição inicial. Eles podem ser verificados em todas as fases da vida, porém representam um problema de saúde mais sério e frequente nas idades avançadas, por causa de seu impacto psicológico e as sequelas físicas ocasionadas. As causas das quedaspodem ser únicas e de clara identificação, ou ainda múltiplas e de difícil individualização( ${ }^{(8)}$.

A ocorrência de queda em um idoso é uma realidade acompanhada de inúmeras consequências, algumas vezes irreversíveis, podendo trazer dificuldades ou impossibilidades para a realização das Atividades de Vida Diária (AVD). Isto acarreta problemas não só para os idosos, mas também para suas famílias e para os serviços de saúde, que mobilizam mais recursos no tratamento e na reabilitação( ${ }^{(2)}$.

No contexto das políticas públicas, existem alguns dispositivos legais que em sua composição voltam-se ao institucionalizado e fazem referência à manutenção da funcionalidade, independência $\mathrm{e}$ autonomia, além de outros atos legais que auxiliam na prevenção de quedas. O Ministério da Saúde instituiu, como uma de suas prioridades no Pacto pela Vida, a saúde do idoso e, nela, a queda como evento sentinela. Dessa forma, encontra-se como indicador de monitoramento do Pacto pela Vida o número de casos de internações hospitalares de idosos por fratura de fêmur ${ }^{(9)}$, um dos principais problemas decorrentes de quedas, dentre outros que podem ser considerados redutores da capacidade funcional do idoso. Assim, cabe ao enfermeiro que atua em ILPI atentar para as questões organizacionais, estruturais e voltadas ao cuidado do idoso, no intuito de manter sua funcionalidade e prevenir quedas.

Nesse sentido, este estudo teve como questão de pesquisa: as ações de melhoria voltadas para uma Instituição de Longa Permanência para Idosos, no Rio Grande do Sul - Brasil, poderão auxiliar o enfermeiro na manutenção da funcionalidade e prevenção de quedas em idosos residentes?

Como objetivo deste estudo, destaca-se: Propor ações de melhoria para uma Instituição de Longa Permanência para Idosos, que auxiliem o enfermeiro na manutenção da funcionalidade e prevenção das quedas dos idosos residentes. 


\section{METODOLOGIA}

Trata-se de recorte de uma pesquisa intitulada "Estado cognitivo e quedas: estudo de correlação em idosos residentes numa ILPI do Rio Grande/RS". A ILPI avaliada localiza-se no Rio Grande do Sul, Brasil, sendo uma instituição centenária, filantrópica, com 65 idosos. Em atendimento aos aspectos éticos, os dados foram coletados após anuência do Comitê de Ética em Pesquisa local com o parecer favorável de número 27/2009.

Este estudo, realizado em setembro de 2009, seguiu os moldes de uma pesquisa avaliativa, entendida como uma ferramenta de identificação de acertos e dificuldades com vistas ao aperfeiçoamento de uma realidade. Nesse tipo de estudo não se trata de examinar comparativamente o ideal (proposto) do que é realizado, mas subsidiar o processo, oferecendo elementos concretos para êxito nas atividades propostas ${ }^{(10)}$.

A construção da ferramenta avaliativa fundamentou-se nas políticas públicas, com intuito de analisar a adequação das mesmas nas ILPI, quanto à manutenção da funcionalidade e à prevenção de quedas. Tal ferramenta objetivou servir de roteiro para o enfermeiro na avaliação dessas instituições e priorizava a organização do serviço, estrutura física e cuidado aos idosos residentes. Sua elaboração, testagem e aplicação foi acompanhada por uma especialista em Gerontologia pela Sociedade Brasileira de Geriatria e Gerontologia. Essa ferramenta foi testada em outra ILPI e, posteriormente, aplicada na ILPI investigada.

Foi selecionado como critério para a elaboração da ferramenta que a mesma tivesse como meta a priorização das condições de produção do conhecimento e, assim, servisse para o aprimoramento do cuidado de enfermagem realizado em ILPI. Tendo como referência a instituição investigada, selecionou-se um avaliador interno (enfermeiro) e outro externo (mestrando), que aplicaram a ferramenta avaliativa, proporcionando um enfoque comparativo entre os dados verificados e o referencial teórico.

A ferramenta avaliativa foi estruturada em quadro com três colunas: na primeira, encontram-se as questões específicas, como organização/estrutura física da ILPI e cuidado ao idoso institucionalizado; na segunda, há espaço destinado às anotações das verificações in loco; na terceira, apresentam-se os dispositivos legais respectivos da fundamentação de cada item a ser avaliado.

A análise dos dados foi realizada tendo como base a ferramenta avaliativa, elaborada a partir das políticas públicas voltadas ao idoso institucionalizado. Procurou-se, também, resgatar as resoluções que regem a atividade do enfermeiro, em especial, as relativas ao cuidado com o institucionalizado, do Estado do Rio Grande do Sul, que foram as Decisões do Coren-RS n. $009 / 2005^{(11)}$ e n. 006/2009 ${ }^{(12)}$. Por fim, a partir dos resultados obtidos com as duas ferramentas avaliativas aplicadas na ILPI, foi possível elaborar propostas de ações voltadas à manutenção da funcionalidade e prevenção de quedas em idosos, tendo como meta a otimização dos cuidados de enfermagem.

\section{RESULTADOS}

Apresentam-se os principais achados na avaliação da ILPI, conforme a disposição dos elementos da ferramenta avaliativa construída: organização, estrutura física e cuidado ao idoso.

Em relação à organização, ao realizar a avaliação da ILPI, encontrou-se um Responsável Técnico (RT) com formação em Serviço Social, com 10 horas semanais de trabalho. Percebeu-se que a ILPI vem buscando atender as exigências de dimensionamento de pessoal, possuindo um médico, um enfermeiro, um fisioterapeuta, um nutricionista, cinco técnicos em enfermagem, e oito cuidadores, para um total de 65 idosos.

Verificou-se que $90 \%$ dos idosos encontram-se na Modalidade I (independência para AVD, que fazem ou não uso de equipamentos de autoajuda) e $10 \%$ na Modalidade II (dependência funcional em qualquer atividade de autocuidado e que necessitam de cuidados específicos). É importante salientar que alguns idosos têm cuidadores particulares e não entram desse quantitativo de trabalhadores da ILPI, sendo cerca de 20 idosos.

Foi possível observar a inexistência de ações periódicas de capacitação e qualificação dos trabalhadores. O enfermeiro da ILPI realiza, durante a jornada de trabalho de 20 horas semanais, atividades de supervisão dos técnicos em enfermagem e dos cuidadores, além de ações de recuperação, proteção e promoção da saúde dos idosos, havendo para tanto um Manual de Normas, Rotinas e Técnicas de Enfermagem atualizado. Outra característica importante na ILPI é a existência de registro de admissão de todos os idosos e do Prontuário do Residente. Ainda, quanto à organização, observouse que a instituição tem um representante no Conselho Municipal do Idoso e apresenta os documentos legais exigidos para que possa manter seu funcionamento.

No que se refere à estrutura física, percebeu-se que a ILPI está edificada em um prédio de grande extensão, com características pavilhonar e vertical, tem um elevador de uso restrito, para segurança e maior co- 
modidade dos residentes, e permanece fechado, sendo preciso que um trabalhador abra e acompanhe o idoso quando for usá-lo. Os pisos internos e externos, bem como as escadas e as rampas, em sua totalidade, não possuem revestimentos antiderrapantes e as escadas não se encontram sinalizadas.

Verificou-se que dormitórios e banheiros são separados por sexo. Os dormitórios coletivos acomodam de uma a quatro pessoas; os banheiros encontram-se nos corredores e apresentam-se escuros, o que torna o acesso noturno difícil. Tais banheiros, em sua maioria, não possibilitam entrada de cadeiras de rodas em todos os sanitários, não existem barras de segurança em todas as bacias sanitárias, as portas apresentam vão livre na parte inferior menor do que $20 \mathrm{~cm}$, e ainda, os pisos apresentam desníveis.

Foi possível observar locais de aglomeração de equipamentos decorativos como mesas centrais, tapetes e sofás, considerados obstáculos para a livre deambulação do idoso residente, podendo tornar-se também, risco para quedas.

Tratando-se do cuidado ao idoso, observou-se que a ILPI desenvolve poucas atividades que estimu- lam a autonomia diária e a capacidade funcional dos residentes; também se verificou a ausência de ações de atividades físicas, como: caminhadas, passeios, participação em grupos de convivência, atividades artísticas como trabalhos manuais ou que estimulem a cognição como oficinas de leitura, embora haja área física e equipamentos disponíveis.

A ILPI tem o Prontuário do Residente, no qual existe espaço reservado para o registro do Processo de Enfermagem; no entanto, verificou-se ausência de sua realização pelo enfermeiro. Existem equipamentos de autoajuda que auxiliam os idosos e permitem a manutenção da capacidade funcional. Os profissionais que atuam na ILPI desconhecem a necessidade de notificar, ao Serviço de Vigilância Sanitária, a ocorrência de quedas com lesão em idosos.

Buscando a melhoria das condições da ILPI, torna-se imprescindível, após essa avaliação, propor algumas ações para a Instituição, tendo como base as políticas públicas, a fim de proporcionar a manutenção da funcionalidade e a prevenção de quedas nos idosos residentes, e que são apresentadas no Quadro 1.

Quadro 1 - Propostas de ações voltadas à manutenção da funcionalidade e prevenção de quedas em idosos institucionalizados. Rio Grande do Sul, 2009

Solicitar que Responsável Técnico (RT) permaneça e cumpra na ILPI a carga horária mínima de $20 \mathrm{hs} / \mathrm{semanais.}$ Desenvolver, com auxílio de estudantes de enfermagem, ações periódicas de capacitação e educação permanente aos profissionais, oferecendo cursos sobre processo de envelhecimento, políticas públicas, dentre outras temáticas. Inserir uma placa de identificação externa na ILPI. Sugere-se: "Instituição de Longa Permanência para Idosos". Manter pisos limpos, secos e não encerados, na impossibilidade da troca dos mesmos.

Reformar pisos externos, extinguindo desníveis.

Utilizar corrimão em todos os corredores e rampas da ILPI.

Nas escadarias e rampas, utilizar sinalização e mecanismos antiderrapantes.

Retirar tapetes e utensílios decorativos de ambientes de maior circulação e que interfiram na deambulação dos idosos residentes.

Colocar luz de vigília nos quartos, corredores e banheiros da ILPI.

Colocar campainha de alarme, pelo menos uma para cada quarto, próxima à cama do idoso mais autônomo. Utilizar barras de segurança em todos os sanitários.

Solicitar auxílio de educador físico para a realização de atividades físicas com os idosos, buscando estimular a autonomia e manutenção da funcionalidade.

Refletir sobre a necessidade de aumento do número de profissionais, considerando o dimensionamento proposto pela RDC $283 / 2005$.

Utilizar, rotineiramente, o Prontuário do Residente e a aplicação de instrumentos voltadas à avaliação das Atividades de Vida Diária dos idosos residentes.

Implementar a Sistematização da Assistência de Enfermagem, principalmente na admissão do idoso, a cada mês, ou em menor tempo, caso necessário.

Estabelecer que todos os episódios de quedas sejam registrados no Prontuário do Residente enotificados à autoridade sanitária. 


\section{DISCUSSÃO}

Constatou-se que a ILPI avaliada atende de forma parcial aos requisitos solicitados pelos documentos legais específicos. A condição de responsabilidade técnica atende parcialmente estes dispositivos legais ${ }^{(3-7)}$. Uma questão que necessita ser discutida na ILPI, visando um melhor cuidado aos institucionalizados, diz respeito ao fornecimento de ações periódicas de capacitação e qualificação dos profissionais, atendendo à legislação vigente.

Na Política Nacional do Idoso encontra-se, na área de educação, a necessidade de inclusão de conteúdos sobre o envelhecimento nos diversos níveis de ensino, objetivando eliminar preconceitos e produzir novos conhecimentos a respeito deste tema. Sugere-se, ainda, a elaboração de programas educativos, nos meios de comunicação, para informar a população sobre o processo de envelhecimento. Outra ação é o estímulo a programas que adotem modalidades de ensino a distância, adequados às condições do idoso ${ }^{(4)}$, dando, assim, a devida importância ao ensino da geriatria e da gerontologia na busca de capacitar os profissionais quanto às especificidades do idoso.

Uma ILPI que atenda as orientações dos dispositivos legais voltados aos institucionalizados, bem como possua profissionais habilitados ao cuidado ao idoso, torna-se mais confiável e segura. Concomitantemente, subsiste a preocupação de manter o ambiente/contexto estrutural adequado, auxiliando, portanto, na manutenção da funcionalidade e minimização da ocorrência de quedas.

A ILPI avaliada possui, em sua organização, um representante legal no Conselho Municipal do Idoso, que é um dos administradores da ILPI, atendendo, portanto, aos dispositivos legais ${ }^{(6-9,11-12)}$. O Conselho do Idoso, seja nacional, estadual ou municipal, segundo o Art. 6 e Art. 7 da Lei n. 8.842/1994 é um órgão permanente, paritário e deliberativo, por isso, compete a ele, a supervisão, o acompanhamento, a fiscalização e a avaliação da Política Nacional do Idoso ${ }^{(4)}$.

Uma característica importante na ILPI é manter o registro de admissão de todos os idosos, bem como possuir o Prontuário do Residente, seguindo, assim, dispositivos legais ${ }^{(3-6,8,13)}$. O Prontuário do Residente é indispensável para prestar um cuidado qualificado aos idosos. Os registros servem para ajustar o atendimento e facilitar o acesso às informações pelos trabalhadores da saúde. Esse prontuário destina-se a registrar os cuidados prestados por cada integrante da equipe multidisciplinar, sendo um documento único, no qual devem ser anotadas todas as infor- mações relativas à saúde de cada idoso ${ }^{(13)}$.

Quanto à estrutura física da ILPI, observou-se que, embora com um adequado aspecto externo, a ILPI não apresenta placa de identificação e é reconhecida devido ao seu trabalho centenário na comunidade. No Estatuto do Idoso, Capítulo IX, encontra-se indicado que a instituição de atendimento ao idoso deve ter identificação externa visível ${ }^{(6)}$. As ILPI deveriam, preferencialmente, ser construções horizontais de caráter pavilhonar, e se edificadas verticalmente, possuir elevadores. A preferência por construções horizontais, as exigências por pisos internos e externos de fácil limpeza e conservação, de padrão uniforme, com ou sem juntas e com mecanismo antiderrapante, são exigências legais ${ }^{(3-4,7,12)}$, e justificam-se diante da manutenção da funcionalidade e na prevenção das quedas em idosos.

Sabe-se que a combinação de fatores intrínsecos como os relacionados a algumas doenças crônicas não transmissíveis - e fatores extrínsecos, que incluem os riscos ambientais, como pisos com brilho excessivo e/ ou encerados, são considerados associações mais importantes para a ocorrência de quedas do que as causas únicas $^{(8)}$. Os locais por onde o idoso circula devem ter, preferencialmente, pisos antiderrapantes, lisos, sem estampas, sem tapetes soltos e livres de objetos que possam confundir a visão/percepção e ocasionar quedas.

A presença de equipamentos decorativos, em especial, o uso de tapetes soltos foi observado em vários locais da ILPI, contrastando com os princípios legislativos, pois favorece a ocorrência de quedas. Estudo realizado no Rio Grande do Sul demonstrou que as quedas foram comuns no ambiente institucional (62,3\%) e o quarto foi o local onde ocorreu o maior número de quedas (23\%). Do total de idosos que sofreram queda, $27,5 \%$ apresentaram alguma fratura ${ }^{(14)}$.

No que se refere ao cuidado aos institucionalizados, deve-se entender como condição fundamental para o exercício profissional do enfermeiro que o mesmo esteja ciente das características do processo de envelhecimento, o que possibilitará prestar um atendimento integral ao residente da ILPI, conservando ao máximo os princípios de autonomia e independência. E também, qualificar a equipe de enfermagem, através da educação permanente em saúde, a fim de capacitá-la para realizar o cuidado ao idoso com maior sensibilidade, segurança, competência e responsabilidade ${ }^{(13)}$.

A ILPI atende a necessidade de existência de equipamentos de autoajuda, como bengalas, cadeiras de rodas e andadores, conforme solicita a legislação específica $^{(4,7,12)}$. Vários aspectos físicos do idoso são 
melhorados com o uso de equipamentos de autoajuda, reconhecidos como Tecnologias Assistivas, levando à prevenção do risco de quedas e fraturas, e à minimização dos déficits relacionados às mudanças características do envelhecimento, como a diminuição da força muscular, da coordenação, do equilíbrio e a instalação de deficiência visual ${ }^{(15)}$. Nesse sentido, é importante que o enfermeiro seja um colaborador na prevenção de quedas, maximizando as potencialidades do idoso, e auxiliando nas medidas ambientais para compensar deficiências, como o uso das Tecnologias Assistivas ${ }^{(16)}$.

Há na ILPI ausência do registro do enfermeiro acerca do Processo de Enfermagem. Esse é, no momento, realizado por estudantes de Enfermagem, durante as atividades práticas de ensino ou de extensão. As principais ocorrências são registradas pelo enfermeiro na folha de anotações dos profissionais da saúde, não atendendo a legislação ${ }^{(12)}$ no que diz respeito à realização do histórico, identificação dos diagnósticos de enfermagem e respectivas prescrições.

\section{CONCLUSÕES}

Com esse estudo se propôs ações de melhoria para uma ILPI localizada no Rio Grande do Sul, Brasil, que auxiliassem o enfermeiro na manutenção da funcionalidade e prevenção de quedas em idosos residentes. Nesse caso, a utilização da pesquisa com enfoque avaliativo parece ter-se mostrado adequada. Como ponto positivo, destaca-se o conhecimento prévio da ILPI e dos idosos residentes pela avaliadora externa, sendo um fator relevante para a avaliação, porque retira o caráter imparcial e meramente julgatório do processo avaliativo. De certa forma, pode-se dizer que a avaliação se deu como um processo participativo.

Como contribuições resultantes desse estudo foram elaboradas ações que podem auxiliar para a manutenção da funcionalidade e para a prevenção de quedas em idosos, e assim propô-las ao enfermeiro que atua em ILPI. Espera-se, ainda, que esta ferramenta avaliativa sirva de ligação entre o Serviço/ILPI e a Academia/ Universidade, para que outras ações estratégicas sejam articuladas e voltadas à melhoria do cuidado de enfermagem ao idoso institucionalizado.

\section{REFERÊNCIAS}

1. IBGE. Instituto Brasileiro de Geografia e Estatística. Perfil demográfico do processo de envelhecimento populacional com base nos resultados da amostra dos censos demográficos 1991 e 2000. Rio de Janeiro: IBGE; 2007.

2. Machado TR, Oliveira CJ, Costa FBC, Araujo TL. Avaliação da presença de riscos para quedas em idosos. Rev Eletrôn Enfermagem. 2009;11(1):8-32.

3. Ministério da Saúde (BR). Lei ${ }^{\circ} .8842$ de 4 de janeiro de 1994 e Decreto Lei no 1948 de 22 de junho de 1996. Dispõe sobre a Política Nacional do Idoso. Diário Oficial da União, Brasília, 1994.

4. Ministério da Saúde (BR). Portaria Interministerial n. 5.153 de 7 de abril de 1999. Institui o Programa Nacional de Cuidadores de Idosos. Diário Oficial da União, Brasília, 1999.

5. Ministério da Saúde (BR). Lei n. 10.741 de $1^{\circ}$ de outubro de 2003. Dispõe sobre o Estatuto do Idoso e dá outras providencias. Diário Oficial da União, Brasília, 2003.

6. Ministério da Saúde (BR). Resolução n. 283, de 26 de setembro de 2005. Aprova o regulamento técnico que define normas de funcionamento para Instituições de Longa Permanência para Idosos. Anvisa. Diário Oficial da União, Brasília, 2005.

7. Araújo MOPH, Ceolim MF. Avaliação do grau de independência de idosos residentes em instituições de longa permanência. Rev Esc Enferm USP. 2007;41(3): 78-85.

8. Fabrício SC, Rodrigues RA, Costa Jr ML. Causas e consequências de quedas de idosos atendidos em hospital público. Rev Saude Publica. 2004;38(1):93-9.

9. Ministério da Saúde (BR). Portaria n.325, de 21 de fevereiro de 2008. Estabelece prioridades, objetivos e metas do Pacto pela Vida para 2008, os indicadores de monitoramento e avaliação do Pacto pela Saúde e as orientações, prazos e diretrizes para a sua pactuação. Diário Oficial União, Brasília, 2008.

10. Belloni I, Magalhães H, Sousa LC. Metodologia de avaliação em políticas públicas: uma experiência em educação profissional. $4^{\mathrm{a}}$ ed. São Paulo: Cortez, 2007.

11. Conselho Regional de Enfermagem do Rio Grande do Sul. Legislação. Decisão do COREN - RS no 009/2005. Dispõe das Normas para definição das atribuições do Responsável Técnico. Porto Alegre; COREN; 2005.

12. Conselho Regional de Enfermagem do Rio Grande do Sul. Legislação. Decisão do COREN - RS n. 006/2009. Dispõe sobre normatizações para o serviço do enfermeiro em instituições de longa permanência 
para idosos. Porto Alegre; COREN; 2009.

13. Santos SSC, Silva BT, Barlem ELD, Lopes RS. O papel do enfermeiro na Instituição de Longa Permanência para Idosos. Rev Enferm UFPE On Line. 2008;2(3):262-8.

14. Gonçalves LG, Vieira ST, Siqueira SV, Hallal PC. Prevalência de quedas em idosos asilados no município de Rio Grande, RS. Rev Saude Publica. 2008;42(5):938-45.

15. Andrade VS, Pereira LS. M. Influência da tecnologia assistiva no desempenho funcional e na qualidade de vida de idosos comunitários frágeis: uma revisão bibliográfica. Rev Bras Geriatr Gerontol. 2009;12(1):113-22.

16. Lenardt, MH; Michel, T; Tallmann, AEC. A condição de saúde de idosas residentes em uma Instituição de Longa Permanência. Cogitare Enferm. 2009;14(2):227-36. 